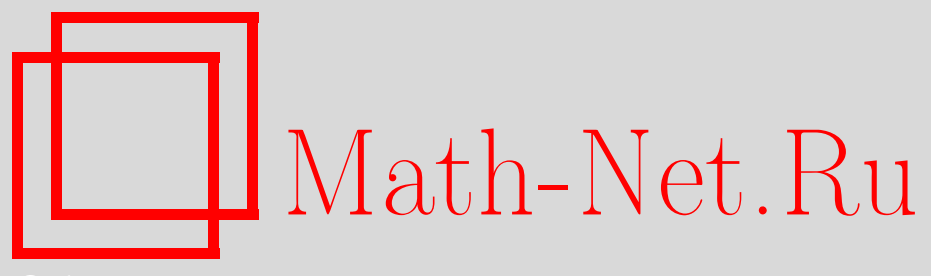

П. А. Крутицкий, Задача Дирихле для уравнения диффузии вне плоских разомкнутых поверхностей, УМН, 2014, том 69, выпуск 4, 183-184

DOI: https://doi.org/10.4213/rm9608

Использование Общероссийского математического портала Math-Net.Ru подразумевает, что вы прочитали и согласны с пользовательским соглашением http: //www . mathnet.ru/rus/agreement

Параметры загрузки:

IP : 3.85 .73 .92

26 апреля 2023 г., 17:23:55

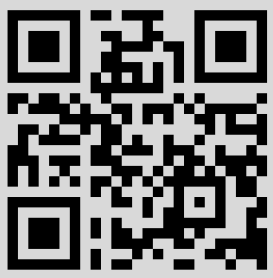




\section{Задача Дирихле для уравнения диффузии вне плоских разомкнутых поверхностей}

\section{П. А. Крутицкий}

В пространстве $\mathbb{R}^{3}$ введем декартовы координаты $x=\left(x_{1}, x_{2}, x_{3}\right)$. Рассмотрим конечное число разомкнутых плоских компактных поверхностей с липшицевой границей, расположенных в плоскости $x_{3}=0$ и содержащих свои предельные точки. Будем предполагать, что эти поверхности не имеют общих точек друг с другом, в том числе не имеют общих граничных точек. Совокупность этих поверхностей обозначим $\Omega$. Тем самым, $\Omega$ - (несвязная) двумерная замкнутая липшицева область на плоскости $x_{3}=0$. Введем соболевские пространства на $\Omega$ :

$$
\begin{gathered}
\widetilde{H}^{-1 / 2}(\Omega)=\left\{u: u \in H^{-1 / 2}\left(\mathbb{R}^{2}\right), \operatorname{supp} u \subset \Omega\right\}, \\
H^{1 / 2}(\Omega)=\left\{u: u=\left.U\right|_{\Omega} \text { для некоторого } U \in H^{1 / 2}\left(\mathbb{R}^{2}\right)\right\}, \\
\|u\|_{\widetilde{H}^{-1 / 2}(\Omega)}=\|u\|_{H^{-1 / 2}\left(\mathbb{R}^{2}\right)}, \quad\|u\|_{H^{1 / 2}(\Omega)}=\min _{\left.U\right|_{\Omega}=u, U \in H^{1 / 2}\left(\mathbb{R}^{2}\right)}\|U\|_{H^{1 / 2}\left(\mathbb{R}^{2}\right)} .
\end{gathered}
$$

Пространства $H^{ \pm 1 / 2}\left(\mathbb{R}^{2}\right)$ и $H_{\mathrm{loc}}^{1}\left(\mathbb{R}^{3}\right)$ вводятся обычным образом [1], [2]. Сформулируем задачу Дирихле для стационарного уравнения диффузии в $\mathbb{R}^{3} \backslash \Omega$.

ЗАДАчА D. Найти функцию $u(x) \in H_{\mathrm{loc}}^{1}\left(\mathbb{R}^{3}\right) \cap C^{2}\left(\mathbb{R}^{3} \backslash \Omega\right)$, удовлетворяющую уравнению

$$
\Delta u(x)-k^{2} u(x)=0, \quad k>0, \quad x \in \mathbb{R}^{3} \backslash \Omega, \quad \Delta=\frac{\partial^{2}}{\partial x_{1}^{2}}+\frac{\partial^{2}}{\partial x_{2}^{2}}+\frac{\partial^{2}}{\partial x_{3}^{2}},
$$

граничному условию $\left.u\right|_{\Omega}=f \in H^{1 / 2}(\Omega)$ и условиям на бесконечности: $u(x)=o\left(|x|^{-1}\right)$, $|\nabla u(x)|=o\left(|x|^{-1}\right)$ при $|x|=\left(x_{1}^{2}+x_{2}^{2}+x_{3}^{2}\right)^{1 / 2} \rightarrow \infty$.

Граничное условие подразумевает, что функция $u(x)$ имеет след на $\Omega$, который удовлетворяет этому условию. Построим решение задачи D. Ищем решение в виде потенциала простого слоя $U[g](x)$ для стационарного уравнения диффузии

$$
\begin{gathered}
u(x)=U[g](x)=\frac{1}{4 \pi} \int_{\Omega} g(y) K(x-y) d y_{1} d y_{2}, \quad K(x-y)=\frac{\exp (-k|x-y|)}{|x-y|}, \\
|x-y|=\left(\left(x_{1}-y_{1}\right)^{2}+\left(x_{2}-y_{2}\right)^{2}+x_{3}^{2}\right)^{1 / 2},
\end{gathered}
$$

с плотностью $g \in \widetilde{H}^{-1 / 2}(\Omega) \subset H^{-1 / 2}\left(\mathbb{R}^{2}\right)$. Функция $U[g](x)$ определена при $x \in \mathbb{R}^{3} \backslash \Omega$.

Из [2] вытекает, что потенциал $U[g](x)$ принадлежит $H_{\mathrm{loc}}^{1}\left(\mathbb{R}^{3}\right)$, имеет след на $\Omega$ : $\left.U[g](x)\right|_{\Omega} \in H^{1 / 2}(\Omega)$ и принадлежит $C^{\infty}\left(\mathbb{R}^{3} \backslash \Omega\right)$ (см. [2; с. 202]). Кроме того, потенциал $U[g](x)$ удовлетворяет условиям на бесконечности. Тем самым, при любой функции $g$ из пространства $\widetilde{H}^{-1 / 2}(\Omega)$ потенциал $U[g](x)$ удовлетворяет всем условиям задачи D, кроме граничного условия. Выберем функцию $g \in \widetilde{H}^{-1 / 2}(\Omega)$ так, чтобы удовлетворить этому условию. Подставляя потенциал $U[g](x)$ в граничное условие задачи D, получаем операторное уравнение

$$
\left.U[g](x)\right|_{\Omega}=f \in H^{1 / 2}(\Omega),
$$

которое изучено в [1]. Из [1] следует, что уравнение имеет единственное решение $g \in \widetilde{H}^{-1 / 2}(\Omega)$ при любой правой части $f \in H^{1 / 2}(\Omega)$. Потенциал $U[g](x)$, построенный на этом решении, удовлетворяет всем условиям задачи D. Таким образом, доказана следующая теорема.

Tеорема 1. Решение задачи D существует и дается формулой (1), где g $\in$ $\widetilde{H}^{-1 / 2}(\Omega)$ - единственное решение уравнения (2), которое однозначно разрешимо в $\widetilde{H}^{-1 / 2}(\Omega)$.

DOI: $10.4213 / \mathrm{rm} 9608$ 
Докажем единственность решения задачи D. Справедлива следующая теорема.

Теорема 2. Задача D имеет не более одного решения.

ДокАзАтельство. Пусть теперь $u(x)$ - решение однородной задачи D. Рассмотрим шар $B_{r}$ достаточно большого радиуса $r$ с центром в начале координат. Считаем, что $\bar{\Omega} \subset B_{r}$ и $\bar{\Omega} \cap \partial B_{r}=\varnothing$. Черта сверху означает замыкание, а $\partial B_{r}-$ сфера, граница шара $B_{r}$. Пусть $\mathbb{R}_{ \pm}^{3}=\left\{x: x \in \mathbb{R}^{3}, \pm x_{3}>0\right\}$, так что $\mathbb{R}_{+}^{3}$ - верхнее полупространство, а $\mathbb{R}_{-}^{3}$ - нижнее полупространство. Положим $B_{r}^{ \pm}=B_{r} \cap \mathbb{R}_{ \pm}^{3}$ и $\partial B_{r} \cap R_{ \pm}^{3}=S_{r}^{ \pm}$. Через $S_{r}$ обозначим пересечение шара $B_{r}$ и плоскости $x_{3}=0$. Под $\partial B_{r}^{ \pm}$понимается граница полушара $B_{r}^{ \pm}$. Поскольку $u \in H_{\mathrm{loc}}^{1}\left(\mathbb{R}^{3}\right)$, то для функции $u(x)$ в $B_{r}^{+}$и в $B_{r}^{-}$ справедлива формула Грина [2; теорема 4.4 , с. 118]:

$$
\begin{aligned}
& \|\nabla u\|_{L_{2}\left(B_{r}^{ \pm}\right)}^{2}+k^{2}\|u\|_{L_{2}\left(B_{r}^{ \pm}\right)}^{2} \\
& \quad=\left(u, \frac{\partial u}{\partial n}\right)_{L_{2}\left(S_{r}^{ \pm}\right)} \mp\left(u,\left(\frac{\partial u}{\partial x_{3}}\right)^{ \pm}\right)_{L_{2}(\Omega)} \mp\left(u,\left(\frac{\partial u}{\partial x_{3}}\right)\right)_{L_{2}\left(S_{r} \backslash \Omega\right)} .
\end{aligned}
$$

Под $n$ понимается внешняя к $B_{r}$ нормаль на $\partial B_{r}$. Под $\left(\partial u / \partial x_{3}\right)^{ \pm}$понимается аналог нормальной производной (след) на $\Omega$ функции $\partial u / \partial x_{3}$ при стремлении к $\Omega$ из $\mathbb{R}_{ \pm}^{3}$. Поскольку $u \in H^{1}\left(\mathbb{R}^{3}\right)$, то след $\left.u\right|_{\partial B_{r}^{ \pm}} \in H^{1 / 2}\left(\partial B_{r}^{ \pm}\right)$функции $u(x)$ на $\partial B_{r}^{ \pm}$при стремлении из $B_{r}^{ \pm}$существует (см. [2; теоремы $3.37,3.38$, с. 102]), и существует след нормальной производной функции $u(x)$ на $\partial B_{r}^{ \pm}:\left.(\partial u / \partial n)\right|_{\partial B_{r}^{ \pm}} \in H^{-1 / 2}\left(\partial B_{r}^{ \pm}\right)$при стремлении к $\partial B_{r}^{ \pm}$из $B_{r}^{ \pm}[2 ;$ с. $116-118]$. Пространства $H^{1 / 2}\left(\partial B_{r}^{ \pm}\right)$и $H^{-1 / 2}\left(\partial B_{r}^{ \pm}\right)$ определяются в [2] и являются двойственными на $\partial B_{r}^{ \pm}$в смысле скалярного произведения в $L_{2}\left(\partial B_{r}^{ \pm}\right)$, следовательно, сумма скалярных произведений в правой части (3) определена. Отметим, что в формуле (3) учтены свойства гладкости функции $u(x)$. Сложим тождества, определяемые формулой (3) для $B_{r}^{+}$и $B_{r}^{-}$, учитывая, что функция $u(x)$ является решением однородной задачи $\mathrm{D}$, а потому удовлетворяет однородному граничному условию: $\left.u\right|_{\Omega}=0 \in H^{1 / 2}(\Omega)$. Тогда получим

$$
\|\nabla u\|_{L_{2}\left(B_{r} \backslash \Omega\right)}^{2}+k^{2}\|u\|_{L_{2}\left(B_{r} \backslash \Omega\right)}^{2}=\left(u, \frac{\partial u}{\partial n}\right)_{L_{2}\left(\partial B_{r}\right)} .
$$

Устремляя $r$ к $\infty$ и принимая во внимание, что функция $u(x)$ удовлетворяет условиям на бесконечности задачи $\mathrm{D}$, находим: $\|\nabla u\|_{L_{2}\left(\mathbb{R}^{3} \backslash \Omega\right)}^{2}+k^{2}\|u\|_{L_{2}\left(\mathbb{R}^{3} \backslash \Omega\right)}^{2}=0$. Поскольку $k>0$, отсюда следует, что $\|u\|_{L_{2}\left(\mathbb{R}^{3} \backslash \Omega\right)}^{2}=0$, т. е. $u \equiv 0$ в $\mathbb{R}^{3} \backslash \Omega$. Таким образом, однородная задача $\mathrm{D}$ имеет только тривиальное решение. В силу линейности задачи $\mathrm{D}$, неоднородная задача D имеет не более одного решения. Теорема доказана.

В заключение отметим, что задача Дирихле для уравнения Лапласа вне плоских разомкнутых поверхностей изучалась в [3].

\section{Список литературы}

[1] П. А. Крутицкий, УМН, 69:1(415) (2014), 171-172; англ. пер.: P. A. Krutitskii, Russian Math. Surveys, 69:1 (2014), 162-164. [2] McLean W., Strongly elliptic systems and boundary integral equations, Cambridge Univ. Press, Cambridge, 2000, xiv+357 pp. [3] A. В. Сетуха, Чucленные методы решения некоторых краевых задач с обобщенными граничными условиями u ux приложения к аэродинамике, Дисс. ... докт. физ.-матем. наук, М., 2003, 372 с.

Павел Александрович Крутицкий

(Pavel A. Krutitskii)

Институт прикладной математики

им. М. В. Келдыша РАН

E-mail: biem@mail.ru
Представлено М. И. Зеликиным Принято редколлегией 05.06.2014 